\title{
Pioderma gangrenosa en cirugía plástica: Comunicación de tres casos
}

\author{
Alex Eulufí $M^{1}$, W ilfredo Calderón $0^{1}$, José Luis Piñeros $B^{1}$, \\ Macarena Silva $C^{2}$, Alvaro Cuadra $C^{1}$, Patricio Léniz $M^{1,3}$, \\ Lyonel $\mathrm{G}$ ómez $\mathrm{S}^{4}$, Christian Eulufí $\mathrm{M}^{\mathrm{a}}$, Daniel Calderón $\mathrm{M}^{\mathrm{b}}$.
}

\section{Pyoderma gangrenosum. Report of three cases in plastic surgery}

Pyoderma Gangrenosum is a rare disease whose etiology is probably autoimmune. We report two males aged 48 and 49 years and one female aged 54 years, with lesions in the right thigh, right leg and in the borders of a surgical incision in the chest. This article gives a brief description of the lesions, tips for diagnosis and help for the initial management and treatment (Rev Méd Chile 2006; 134: 339-44).

(Key words: Pyodema gangrenosum; Skin diseases, vascular)

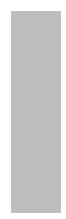

\footnotetext{
Recibido el 1 de abril, 2005. Aceptado el 4 de agosto, 2005.

${ }^{1}$ Servicio de Cirugía Plástica y Quemados del Hospital del Trabajador de Santiago. ${ }^{2}$ Servicio de Medicina Interna, Hospital San Borja-Arriarán. ${ }^{3}$ Escuela de Medicina, Universidad Diego Portales. ${ }^{4}$ Servicio de Cirugía, Hospital Militar de Santiago.

aAlumno de Medicina, Universidad Andrés Bello.

bAlumno de Medicina, Universidad Mayor. Santiago de Chile.
}

$\mathrm{L}$ a pioderma gangrenosa (PG) es una patología principalmente cutánea de características inflamatoria, reactiva, no infecciosa, no tumoral ${ }^{1}$. Es una alteración con un amplio espectro en cuanto a su presentación. Puede variar desde una úlcera moderada, crónica y localizada, hasta una enfermedad multisistémica asociada principalmente a patologías autoinmune de rebelde respuesta a tratamiento a las cuales se asocia su patogenia ${ }^{2}$.

Correspondencia a: Dr. Alex Eulufí Muñoz. Servicio de Cirugía Plástica y Quemados, Hospital del Trabajador de Santiago. Avda. Vicuña Mackenna 200, Providencia. Fax: 6853518. E mail: aeulufi@gmail.com
Su descripción clásica es la presencia de una o más lesiones ulceradas cutáneas, dolorosas, de aspecto infeccioso, secreción purulenta, de bordes irregulares y con una desmesurada respuesta al trauma local (curaciones, aseos quirúrgicos) denominado patergia 3,16 . Debido a la agresividad del cuadro, este puede ser fácilmente confundido con una fasceítis necrotizante del tipo Estreptococo ßhemolítica o infección por anaerobios como el Clostridium perfingens. Se describen alteraciones en la inmunidad celular y humoral además de alteraciones en la función de los polimorfos nucleares ${ }^{4}$.

El diagnóstico es básicamente por presunción y descarte de otras hipótesis etiológicas, incluso a veces asociado a una prueba terapéutica basada 
en el uso de corticoides intralesionales ${ }^{5}$. El laboratorio muestra un patrón infeccioso, donde priman la leucocitosis y las formas juveniles, con proteína C reactiva (PCR) elevada y cultivos de herida o hemocultivos negativos ${ }^{5}$. El estudio anatomopatológico informa generalmente tejido inflamatorio inespecífico ${ }^{6,7}$, aunque algunos autores describen vasculitis cutánea, principalmente necrotizante ${ }^{8,9}$. No existe ningún marcador diagnóstico específi$\mathrm{CO}^{10}$.

Su escasa frecuencia hace que centros de referencia no informen más de dos o tres casos nuevos al año. A modo de ejemplo, la Clínica Mayo en 53 años logró recolectar sólo 180 casos $^{11}$.

La primera descripción del cuadro la realizaron Brunsting et al, en 1930, presentando cinco pacientes con erupciones cutáneas, de los cuales cuatro presentaban colitis ulcerosa. Lo denominó como "pioderma gangrenosa" debido a que el autor pensó se trataba de una infección estreptocócica que desarrollaba una gangrena cutánea ${ }^{12}$. En cuanto a las publicaciones nacionales indexadas, destaca el informe de sólo un caso clínico publicado en la Revista Médica de Chile ${ }^{28}$. En cirugía plástica, existen a la fecha dos presentaciones orales de esta patología, la primera por parte del Dr. Mario Godoy S, en el Congreso de Cirugía Plástica del Cono Sur en Santiago de Chile en 1999 y la otra, por parte de los autores de esta publicación en el Congreso Chileno de Cirugía en 2001. Por lo tanto, este reporte es la primera serie clínica publicada en relación a pioderma gangrenosa y cirugía plástica, tanto estética como reconstructiva.

A pesar de lo errado de la presunción infecciosa del cuadro, la descripción inicial de la patología acertó significativamente en cuanto a la relación patológica de la PG con otras patologías. Las más habituales son las enfermedades inflamatorias intestinales (50\%), la artritis reumatoides (37\%), gammapatía monoclonal (10\%) y leucemia mieloide aguda o crónica (7\%). Esta incidencia varía en relación al subtipo de PG, la cual puede ser pustular, vegetativa, ulcerosa y bulosa.

Por lo tanto, esta patología puede transformarse en una terrible carga para el tratante, especialmente si se presenta en relación a cirugía electiva, y peor aún si es del tipo estético.

El objetivo de este trabajo es llamar la atención sobre este infrecuente y raro cuadro clínico y dar pautas para su diagnóstico y tratamiento.

\section{Casos cLínicos}

Caso 1. Hombre de 48 años, sin patología mórbida de base. Antecedente de haber sufrido cinco meses previo al ingreso hospitalario un flegmón en pierna derecha, aparentemente secundario a un loxocelismo cutáneo, tratado. Como parte del tratamiento se le realizó un injerto dermo epidérmico (IDE), en la zona ahora comprometida.

Fue derivado para manejo de ulceraciones y bordes necróticos en la zona injertada sin respuesta a las continuas curaciones ni antibioterapia.

Al ingreso se constató una herida con bordes necróticos, profundo centro ulcerado y secreción de aspecto purulento, en tercio medio cara lateral del muslo derecho. El laboratorio mostró leucocitosis de 30.000; V.H.S. $100 \mathrm{~mm} / \mathrm{hr}$. El paciente se encontraba en buenas condiciones generales con intenso dolor en relación a la zona cruenta. Fue sometido a aseo quirúrgico y toma de cultivos de tejido por el equipo de cirugía plástica. A las 48 h de la cirugía la lesión mostraba una evidente expansión en diámetro y profundidad. El laboratorio se mantenía con patrón séptico, aunque la tinción de Gram y los cultivos cuantitativos de tejido eran negativos.

Al cuarto día del ingreso fue sometido a un segundo aseo quirúrgico, debido al aspecto séptico de la lesión. Nuevamente la tinción de Gram y los cultivos de tejido fueron negativos. La lesión no mostró mayor deterioro, aunque tampoco notoria mejoría en los días posteriores. Al décimo sexto día del ingreso se decidió asear por tercera vez la lesión y realizar un IDE, utilizando como zona dadora el tercio medio cara medial del muslo izquierdo. Al quinto día postoperatorio, la zona dadora presentaba exudado purulento, lesiones crateriformes con rebordes solevantados. Se decidió realizar un nuevo aseo quirúrgico, en el cual destaca extensa cantidad de tejido de aspecto cerebroideo y necrótico tanto en la zona injertada como en la zona dadora. A los cuatro días de realizada esta última intervención, la zona dadora mantenía bordes necróticos y solevantados. Las biopsias obtenidas eran inespecíficas sin diagnóstico anatomopatológico. El laboratorio persistía con leucocitosis y predominio de las formas juveniles y VHS elevada. En relación a evaluación por equipo de medicina interna, se realizó un mielograma bajo sospecha de enfermedad mieloproliferativa, el cual tampoco orientó al diagnóstico. 


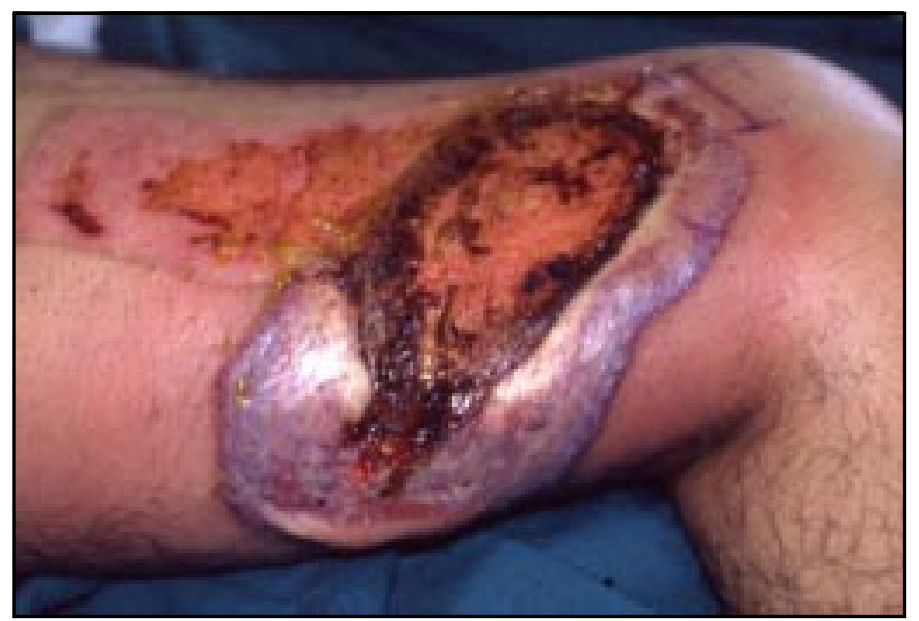

Figura 1. Presentación clásica de PG. Bordes solevantados de aspecto purulento, necrosis y úlceras confluentes centrales.

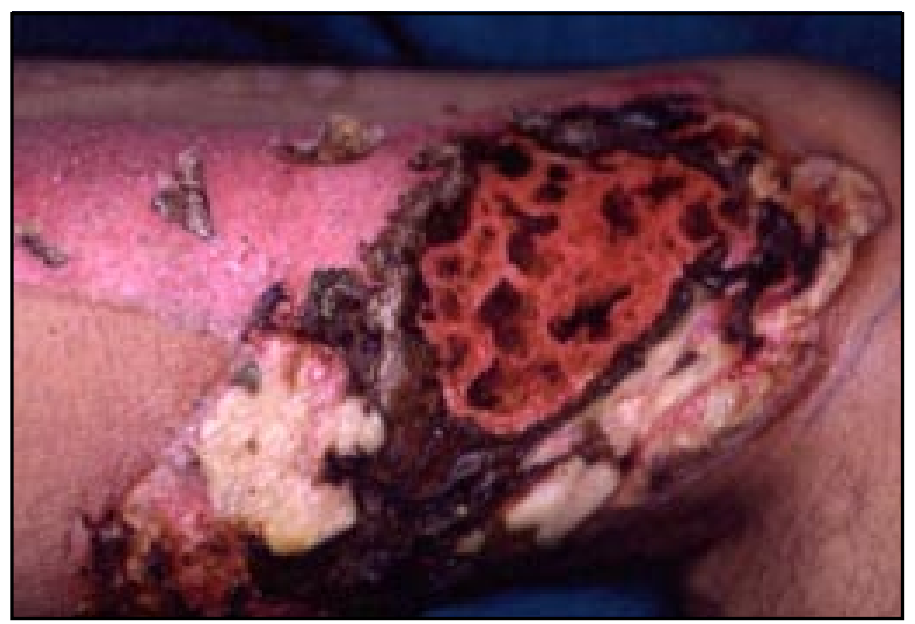

Figura 2. La lesión se circunscribe con 5 días de infiltración y uso sistémico de corticoides (Caso 1).

Se solicitó evaluación por dermatología por probable pioderma gangrenosa. En relación a historia clínica y características de las lesiones, se estableció el diagnóstico presuntivo y se inició terapia esteroidal de manera empírica. El tratamiento consistió básicamente en el uso de corticoides subcutáneos perilesional y la posterior inmunosupresión sistémica. A las $48 \mathrm{~h}$ de tratamiento la favorable evolución de las lesiones era evidente. Se adicionó oxígenoterapia hiperbárica buscando mitigar el dolor, lo cual no fue bien tolerado por el paciente, razón por la cual se suspendió.
A las tres semanas de iniciado el tratamiento el progreso era satisfactorio con epidermización completa de la zona injertada y dos tercios de la zona dadora. A los cincuenta días de iniciado el tratamiento esteroidal se obtuvo epidermización y remisión completa de las lesiones.

Caso 2. Hombre de 49 años, hipertenso arterial sin diagnóstico hasta el ingreso. Presentaba historia de veinte días de evolución con herida pretibial derecha en relación a contusión con portón de fierro. La lesión tenía bordes necróticos y un halo inflamatorio de 
aspecto infeccioso. Al tercer día hospitalizado, debido a la tórpida evolución de la lesión, se realizó escarectomía y se inició antibioterapia empírica (clindamicina y cefuroximo). Luego de la cirugía reaparecieron los bordes necróticos, el halo inflamatorio y se adicionó la presencia de secreción turbia, espesa sin mal olor. Al cuarto día postoperatorio se realizó un segundo aseo quirúrgico. Al día siguiente del segundo acto quirúrgico apareció una lesión pequeña maleolar ipsilateral con las mismas características de la ubicada en la zona pretibial. Ambas lesiones fueron incrementando su tamaño y profundidad, manteniendo secreción de aspecto purulento y bordes necróticos. Los hemocultivos, la tinción Gram y los cultivos de tejido permanecían negativos. Manteniendo leucocitosis, PCR y VHS elevadas. Se decidió realizar un tercer aseo quirúrgico. A las 48 h de la tercera cirugía, ambas lesiones presentaban úlceras sucias con lecho pálido, fibrina y bordes necróticos. Se realizó un cuarto aseo en pabellón y la colocación de una curación con sistema de aspiración negativa continua (Vaccum assisted Closure -VAC), sin éxito. El resultado de la biopsia de tejido obtenido informó hallazgos inespecíficos. El paciente completaba dos semanas de antibióticos y cuatro aseos quirúrgicos sin respuesta favorable. Debido a la sospecha clínica se solicitó la evaluación por dermatología, que confirmó el diagnóstico presuntivo de pioderma gangrenosa. Se inició tratamiento asociado de betametasona inyectable por vía subcutánea rodeando las lesiones e inmunosupresión sistémica con micofenolato mofetil. Se asociaron 40 sesiones de oxígeno terapia hiperbárica para manejo del dolor y favorecer la epidermización. A los 22 días de terapia inmunosupresora, el paciente presentó insuficiencia renal secundaria al uso del micofenolato mofetil, por lo cual se suspendió e inició el uso de prednisona sistémica con buena tolerancia y evolución.

El último control del paciente se realizó a los cinco meses de iniciado el tratamiento, con total epitelización de las lesiones sin recidiva y sin secuela de la insuficiencia renal la cual se recuperó íntegramente.

Caso 3. Mujer de 54 años, sana, sometida a reducción mamaria bilateral por gigantomastia. El tercer día postoperada inició cuadro febril de $38^{\circ} \mathrm{C}$ axilar, con herida operatoria limpia y seca. Resto del examen físico en límites normales. El laboratorio mostró PCR cien veces el valor normal, VHS 60 y GB 10.800. Al cuarto día postoperada persistía febril y con zonas congestivas en los bordes de las heridas operatorias, sin cambios en el laboratorio. Orina y hemocultivos negativos. Se solicitó evaluación por infectología planteándose una neumopatía aspirativa como causa del cuadro clínico y se inició tratamiento antibiótico con moxifloxacino. Al quinto día del postoperatorio el eritema perilesional era mayor y se adicionó una colección de aspecto purulento en la mama izquierda. Se realizó aseo quirúrgico y toma de cultivos y se agregó vancomicina al tratamiento antibiótico previamente señalado. Al día seis la paciente se encontraba afebril sin dolor y en la tinción de Gram no hubo desarrollo bacteriano, sólo destacaba la presencia de gran cantidad de polimorfonucleares, por lo que se planteó el diagnóstico de pioderma gangrenoso. Al octavo día postoperatorio la paciente presentaba eritema intenso de las mamas, tres úlceras exudativas y fiebre intermitente. Laboratorio con VHS 100 y PCR de doscientas veces su valor. Nuevamente fue evaluada por equipo de infectólogos, quienes cambiaron el moxifloxacino por cefipime y se adicionó amikacina. Al mismo tiempo fue evaluada por dermatología quienes plantearon infección por estreptococo, pioderma gangrenosa o desarrollo de úlcera herpética. Al décimo día postoperatorio, debido a la progresión del cuadro, se decidió el uso de corticoides tópicos y sistémico en forma empírica. Se obtuvo una biopsia con hallazgos sugerentes de pioderma gangrenoso en el contexto clínico de la paciente. Al día quince postoperatorio la paciente presentaba una clara regresión del cuadro con importante cicatrización de las úlceras. Se suspendió la antibioterapia y se mantuvo el tratamiento esteroidal. Al mes se obtuvo cicatrización secundaria por completo.

\section{DisCUSIÓN}

Se desconoce la real incidencia de la pioderma gangrenosa ni su etiología, aunque las mayores sospechas patogénicas apuntan a una exagerada respuesta autoinmune como desencadenante de las lesiones secundaria a la agresión quirúrgica o de otro tipo ${ }^{12}$. No hay datos para suponer que sea más frecuente en hombres o en mujeres ${ }^{5}$, aunque hay estudios que muestran mayor ocurrencia en mujeres jóvenes especialmente el grupo etario entre los 30 y 50 años $^{13}$, sin embargo, también puede presentarse en el grupo pediátrico ${ }^{11,14}$. 


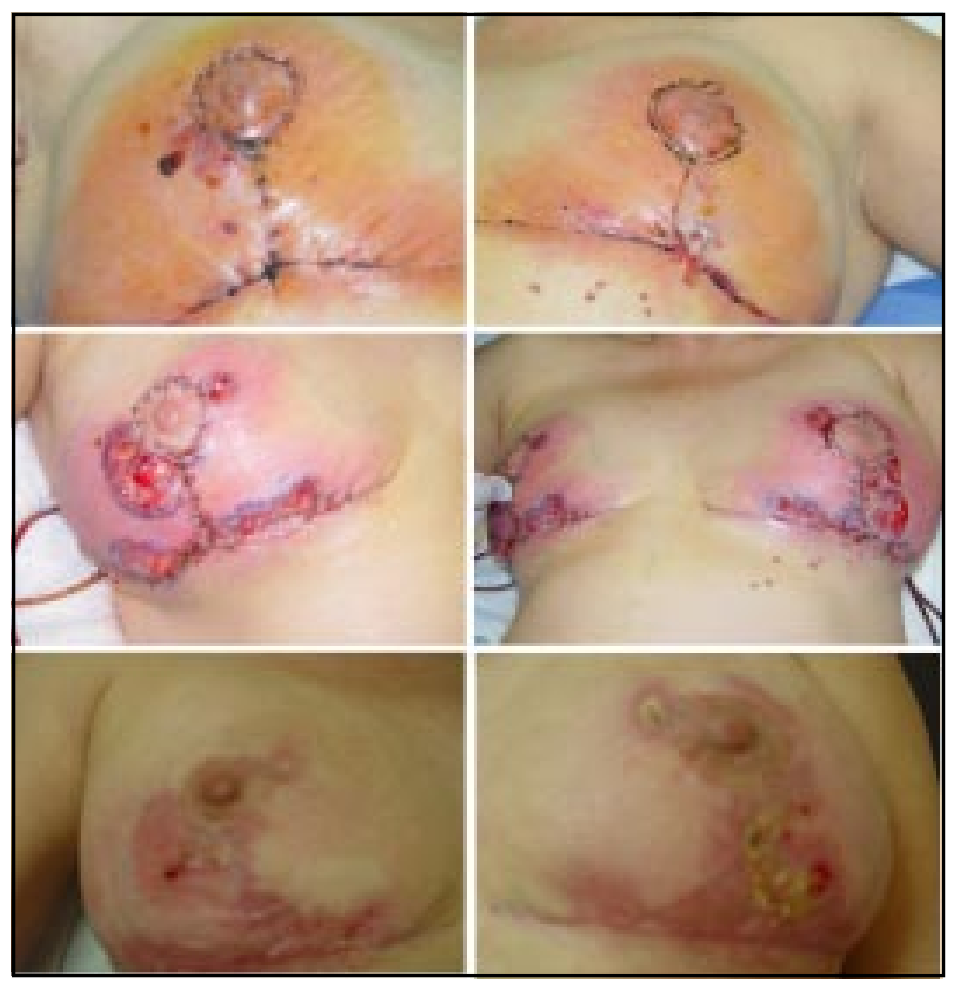

Figura 3. Cuadro fotográfico que demuestra la evolución clínica desde el segundo día post operatorio hasta su resolución completa. (Caso 3).

Se reconoce un patrón medianamente uniforme caracterizado por lesiones ulceradas con bordes necróticos y secreciones de aspecto purulento, sin mal olor, generalmente con estudio bacteriológico negativo $^{15,16}$, de no coexistir sobreinfección. El estudio histopatológico es inespecífico, siendo lo más frecuente encontrar una dermatosis neutrofilica con características de patrón vasculítico leucocitoclástico o linfocítico de hallazgos absolutamente inespecíficos, por lo cual el diagnóstico debe apoyarse en la evolución clínica y la sospecha de la enfermedad ${ }^{17}$.

El tratamiento está basado en la esteroide terapia local $^{18}$ y sistémica ${ }^{18,1,5}$ requiriendo incluso la utilización de inmunosupresores, siendo la ciclosporina la más utilizada y con mejores respuestas clínicas $^{19,20,22}$. Existe experiencia con el uso de FK506 (Tacrolimus) $^{18,20,21,23}$ como potente agente inmunosupresor anticélulas $\mathrm{T}$ el cual también ha mostrado buenas respuestas. La literatura describe, aunque no con mucha consistencia, la inclusión de la oxigenoterapia hiperbárica con el fin de favorecer la epitelización de las zonas ulceradas ${ }^{24}$, aunque empí- ricamente se ha evidenciado que su mayor utilidad se relaciona con la disminución del dolor como sucedió en uno de nuestros casos. También está descrito el uso de radioterapia local sobre la lesión, la plasmaféresis y el uso de interferón con variados grados de satisfacción en cuanto a la respues$\mathrm{ta}^{18,25,26}$.

Cuánto tiempo mantener el tratamiento inmunosupresor también es una incógnita, se describe iniciar progresivamente el retiro del tratamiento una vez que se alcance la total remisión de las zonas afectadas. Las recurrencias intermitentes son frecuentes y no se ha logrado demostrar que el tratamiento más prolongado las prevenga ${ }^{24}$.

Someter a estos pacientes a un nuevo acto quirúrgico electivo es una aventura de la cual nadie puede predecir el resultado. Es además obligación del tratante educar al paciente en relación a la prevención de futuros traumas. Debido al alto índice de recurrencia y la morbilidad asociada a esta patología se debe mantener un seguimiento a largo plazo de estos pacientes ${ }^{27}$. 


\section{REFERENCIAS}

1. Wines N, Wines M, RYMAN W. Understanding pyoderma gangrenosum: a review. General Med 2001; 27 : 6.

2. Powell FC, Schroetr AL, Su WPD, Perry hO. Pyoderma gangrenosum: a review of 86 cases. Q J Med 1985; 55: 173.

3. Hevia H, Suárez J, Vergera MT. Pioderma gangrenoso peri ileostómico: caso clínico. Rev Méd Chile 2004; 132: 747-9.

4. Vignon-Penamen M, Wallach D. Cutaneous manifestation of neutrophilic disease: a study of seven cases. Dermatologica 1991; 183: 255-64.

5. Frank C, Powell, FrCPI WP, Daiel Su MD, Harold O, Perry MD. Continuing medical education. Pyoderma gangrenosum: Classification and management. Journal of the American Academy of Dermatology, Volume 34, number 3, march 1996, 395-409.

6. Percival GH. Pyoderma gangrenosum: The histology of the primary lesion. Br J Dermatol 1957; 69: 130-6.

7. VAN DER Sluis I. Two cases of pyoderma (erthyma) gangrenosum associated with the presence of an anormal serum protein- beta 2A (paraprotein): with a review of the literature. Dermatologica 1966; 132: 409-24.

8. Thompson DM, Main RA, Beck JS, Albert-Recht F. Studies on a patient with leukocitoclastic vasculitis "pyoderma gangrenosum" and paraproteinemia. Br J Dermatol 1973; 88: 117-25.

9. Su WPD, Schroeter Al, Perry HO, Powell FC. histophatologic and inmunopathologic study of pyoderma gangrenosum. J Cutan Pathol 1986; 13: 323-30.

10. Crowson N, Mihm Jr M, Magro CM. Pyoderma gangrenosum: a review. J Cutan Pathol 2003; 30: 97-107.

11. Powell FC, Perry HO. Pyoderma gangrenosum in childhood. Arch Dermatol 1984; 120: 757-61.

12. Brunsting LA, Goeckenman WH, O'Leary PA. Pyoderma gangrenosum: clinical and experimental observations in five cases occurrig in adults. Arch Dermatol 1930; 22: 655.

13. Crowson AN, Magro CM. The pathology of cutaneous drug eruption. Curr Probl Dermathol 2002; 14: 117.
14. Glass AT, Bancila E, Milgraum S. Pyoderma Gangrenosum in infancy: the youngest reported patient. J Am Acad Dermatol 1991; 25: 109-10.

15. Callen JP, Woo TU. Vesicopustular eruption in a patient with ulcerative colitis. Pyoderma gangrenosum. Arch Dermatol 1985; 121: 399-402.

16. Magro CM, Crowson AN, Minm MC. Cutaneous manifestations of gastrointestinal disease. In: Elder DE, Johnson BE, Jawosky C, Elenitsas R, eds. Lever's histopathology of the skin. $8^{\text {th }}$ edn. Philadelphia: JB Lippincott, 1997; 353.

17. Hurwitz RM, Hareman JM. The evolution of pyoderma gangrenosum: a clinicopathologic correlation. Am J Dermatophatol 1993; 15: 28-33.

18. CHow RKP, Ho VC. Treatment of pyoderma gangrenosum. J Am Acad Dermatol 1996; 34: 1047.

19. Elgart G, Stover P, Larson K et al. Treatment of pyoderma gangrenosum with cyclosporina: results in seven patients. J Am Acad Dermatol 1991; 24: 83.

20. Matis WL, Ellis CN, Griffiths CEM, Lazarus GS. Treatment of pyoderma gangrenosum with cyclosporina. Arch Dermatol 1992; 128: 1060.

21. RAPINI RP. Cytotoxic drugs in the treatment of skin disease. Int J Dermatol 1991; 30: 313.

22. Faulds D, Goa KL, Benfield P. Cyclosporin: a review of its pharmacodynamics and pharmacokinetics properties and therapeutics use in inmunoregulatory disorders. Drugs 1993; 45: 953-1040.

23. Ruzicka T, Assman T, Hamey B. Tacrolimus: The drug for the future of the millennium. Arch Dermatol 1999; 135: 574-80.

24. Powell F, O'Kara M. Management of pyoderma gangrenosum. Dermat Cli 2002(20): 2.

25. Kamiuska R, Ikaheimo R, Hollmen A. Plasmapheresis and cyclophosphamide as successful treatments for pyoderma gangrenosum. Clin Exp Dermatol 1999; 24: 81.

26. Beurey J, Canton PH, Weber M et al. Pyoderma gangrenosum cervical: traitement par échanges plasmatiques. Ann Dermatol Venereol 1982; 109: 455-9.

27. Von Den Driesch P. Pyoderma gangrenosum: A Report of 44 cases with follow-up. Br J Dermatol 1997; 137: 1000-5.

28. Hevia H, Suárez J, Vergara MT. Pioderma gangrenoso periileostómico: caso clínico. Rev Méd Chile 2004; 132: 747-9. 